\title{
Los inicios de la automatización de bibliotecas en México. Juan Voutssás Márquez - 2019 - Reseña Bibliográfica
}

\author{
The beginnings of library automation in Mexico \\ Juan Voutssás Márquez - 2019 - Reseña Bibliográfica \\ Alexy Amanda Paz Meneses \\ Licenciada en Informática. Especialista en Finanzas \\ Maestranda en Estudios Latinoamericanos del Centro de Estudios e Investigaciones Latinoamericanas \\ CEILAT de la Universidad de Nariño \\ Email: alexy.paz@gmail.com
}

Doi: https://doi.org/10.22267/rceilat.214849.101

\section{Resumen}

El presente texto reseña la obra del Dr. Juan Voutssás Márquez titulado "Los inicios de la automatización de bibliotecas en México", publicado en línea en el año 2019; se hace un recorrido sobre la estructura general del texto destacando datos y situaciones que permiten comprender las intenciones del autor. Se enfatiza en los conceptos que ilustran significativamente el proceso de automatización de bibliotecas y se sitúa la obra como un recurso bibliográfico indispensable en el campo de la bibliotecología y de las ciencias de la información.

Palabras Clave: Automatización; Ciencias de la Información; Tecnología; Desarrollo; Bibliotecas.

\begin{abstract}
This text reviews the work of Dr. Juan Voutssás Márquez entitled "The beginnings of library automation in Mexico", published online in 2019; a tour of the general structure of the text is made highlighting data and situations that allow understanding the author's intentions. Emphasis is placed on concepts that significantly illustrate the process of library automation and the work is positioned as an indispensable bibliographic resource in the field of library science and information science.
\end{abstract}

Keywords: Automation; Information Sciences; Technology; Development. 


\section{Introducción}

La obra del Dr. Juan Voutssás Márquez" "Los inicios de la automatización de bibliotecas en México" publicada en línea en el año 2019 se incorpora a la bibliográfica especializada en el campo de las ciencias de la información y de la bibliotecología. Esta publicación, hace parte de la colección de Tecnologías de la Información del Instituto de Investigaciones Bibliotecológicas y de la Información de la Universidad Nacional Autónoma de México UNAM.

El Dr. Voutssás, según lo manifiesta en el prólogo parte de algunas ideas sobre la automatización de las bibliotecas en México presentadas en la conmemoración de los cuarenta y cinco años de estudios universitarios en Bibliotecología en el año 2001, manifiesta además que si bien en dicha ocasión su texto a modo de ensayo o memorias no contaba con fuentes documentales que soportan sus apreciaciones, en esta oportunidad, sucede lo contrario, su obra está fuertemente documentada e ilustrada y pretende a través de sus líneas comunicar a sus lectores sobre la problemática, los contextos y los retos que han enfrentado las bibliotecas a través del tiempo.

1 Juan Voutssás Márquez - "Ingeniero Industrial especializado en sistemas de la Universidad Nacional Autónoma de México UNAM, Maestro en Bibliotecología y Doctor en Bibliotecología y estudios de la información, catedrático e investigador titular, con notable experiencia y recorrido teórico y práctico en el campo de las ciencias de la Información y la tecnología" http://iibi.unam.mx/voutssasmt/voutssas_principal.html. Fecha de consulta: 10 junio 2020.
El texto se encuentra dividido en doce (12) partes, diez (10) de las cuales corresponden a la temática central de su disertación. En las primeras cinco, el autor hace un recorrido general sobre los contextos sociales y las problemáticas que dieron lugar a los primeros indicios de los procesos de automatización en las bibliotecas; en adelante, el autor se centra en lo acontecido particularmente en México y para ello ha desarrollado sus ideas de forma fragmentada dependiendo principalmente de la época en que suceden los hechos que marcan la incursión de la tecnología en las bibliotecas. La parte once (11) contiene las conclusiones y la doce (12) contiene las referencias bibliográficas utilizadas.

\section{La automatización de bibliotecas - Contexto general}

La primera parte del texto que desarrolla el Dr. Voutssás, pretende centrar al lector en el concepto de automatización como aquel "dispositivo -mecánico, eléctrico, electrónico, etcétera- para minimizar o sustituir en un proceso a un operador humano" (Voutssás Márquez, 2019, pág.1), permitiendo al lector la posibilidad de asociar las bibliotecas con las tecnologías de la información y los dispositivos tanto no computacionales como computacionales.

En este sentido el autor hace un balance de la tecnología usada en las bibliotecas desde los primeros índices en tabletas de arcilla o los registros de la biblioteca de Alejandría en pinakes, hasta los catálogos de bibliotecas eu- 
ropeas en el siglo XVI. Más adelante, asocia con tecnología los sistemas de clasificación como los del Vaticano, Brunet, Melvil Dewey, Cutter, Ranganathan entre otros; argumenta que la tecnología puede ser o no evidentemente física, por lo que es cuidadoso aclarando que toda tecnología no implica automatización, es decir, la tecnología no se asume unívocamente como un instrumento o dispositivo tangible sino también como el conocimiento aplicado, por ello los distintos métodos de clasificación documental propuestos resultan ser buenos ejemplos para entenderlos también como tecnología aplicada en bibliotecas.

Para ejemplificar el concepto de automatización, el autor se refiere a dispositivos que en la actualidad pocos conocen y para otros resultan ser inauditos, como por ejemplo: la "Rueda de Libros" inventada en 1588 como instrumento de lectura, las tarjetas catalográficas en sus diferentes versiones, sistemas de índices, cajoneras desplegables, máquinas de escribir que sirvieron para agilizar la elaboración de tarjetas de catálogos que se hacían a mano, máquinas que fueron innovándose y algunas fabricándose exclusivamente para bibliotecas; afirma también la existencia de otras máquinas basadas en tarjetas perforadas que fueron perfeccionándose de tal forma que las bibliotecas empezaron a utilizar estos conceptos en sus procesos; dichos artefactos o dispositivos que fueron evolucionando permitían a las bibliotecas llevar a cabo las actividades más importantes del momento: almacenar y recuperar información documental.
Se puede inferir que las tarjetas tuvieron gran éxito, sin embargo, el texto menciona otras tecnologías que cobran relevancia; la fotografía, por ejemplo, llevó a la idea de pretender reducir y crear micro imágenes, además, con el tiempo y de la mano de grandes visionarios, la idea de los micro formatos prosperó como método para la preservación de documentos. Como bien se sabe, con la revolución tecnológica de finales del siglo XIX e inicios del siglo XX la información crece vertiginosamente, razón por la cual los micro formatos resultaban ser una tecnología bastante interesante para uso de la biblioteca, es así como la microfilmación fue un excelente soporte para preservar y difundir colecciones documentales, años más tarde, surge un nuevo concepto que combina la tarjeta perforada y los microformatos: la "tarjeta con ventana" y posteriormente el autor se refiere a las microfichas.

Se resalta además, que otras máquinas como el teleautógrafo hicieron lo propio en las bibliotecas permitiendo enviar a distancia información escrita a mano; y para automatizar los préstamos, el autor describe artefactos como la prensa Dickman, la máquina Gaylord, el Cardinner.

Con el desarrollo de nuevas tecnologías la información se traslada a transparencias o diapositivas y con ellas surgen los "carruseles" para su proyección, al combinarse con los casetes de audio o música pronto las bibliotecas incorporaron entre sus propósitos la producción de material 
audiovisual para la creación de tutoriales, talleres etc. Así sucesivamente recorre de manera narrativa gran variedad de inventos tecnológicos no computacionales que a través de imágenes que sustentan dichas descripciones transporta a sus lectores a un espacio y tiempo hoy irreconocible.

De esta primera parte se puede destacar además el papel de las empresas que vieron en la tecnología una manera de agilizar los procesos en las labores de la biblioteca y una forma de fortalecer su labor comercial. La tecnología descrita pretende facilitar y hacer más eficiente el trabajo del bibliotecario que en principio era manual y que por ende toma mucho tiempo, implica costos y mucha más dedicación. Una constante en los esfuerzos realizados, consiste en agilizar el proceso de recuperación de la información, concepto que ha dado lugar a diversos y variados estudios dentro de las Ciencias de la Información, uno de los puntos clave del campo disciplinario.

En la segunda parte, el autor describe las tecnologías computacionales que surgen en Estados Unidos y Gran Bretaña en la primera mitad del siglo $\mathrm{XX}$ durante la segunda guerra mundial, destaca en sus apreciaciones a la Computadora e Integradora Numérica Electrónica (ENIAC) y a las computadoras Bombe y Colossus que apoyaron a fuerzas militares y de inteligencia, más adelante, para agilizar procesos relacionados con el Censo de Estados Unidos surge UNIVAC I considerada la primera computadora adquirida comercialmente; además, se mencio- na que muchas empresas del sector industrial, financiero $\mathrm{y}$ de servicios vieron las ventajas de estos equipos y comenzaron a adquirirlos.

El autor es minucioso en describir los detalles de funcionamiento, costos de adquisición, renta y tamaño de estas máquinas, señala que para la época, su potencial para el cálculo numérico era un aspecto bastante conveniente para ciertas actividades de tipo científico, administrativo, entre otras; las características de tamaño, costo y velocidad de procesamiento de la primera mitad del siglo XX son inconcebibles en la actualidad. El acceso a ellas era altamente limitado, no cualquiera podía adquirir una de estas máquinas, los recursos económicos que se requerían eran exorbitantes.

Las potencialidades de estas máquinas, menciona el autor, hacen posible que instituciones universitarias también quisieran adquirirlas, aunque los costos fueran elevados, sin embargo, manifiesta que la Universidad Nacional Autónoma de México (UNAM) en el año de 1956 se planteó la idea de adquirir una de estas para el procesamiento automatizado de datos, claro está, rentada y con el fin de resolver problemas de tipo científico en las áreas de las matemáticas y la física. Finalmente según las fuentes del Dr. Voutssás, fue en el año 1958 cuando llega oficialmente el primer equipo computacional a la UNAM, más precisamente al Centro de Cálculo Electrónico (CCE), esta sería la primera computadora destinada a la academia e investigación en toda Iberoamérica. 
Para la época, las bibliotecas requerían dar solución a ciertos problemas producto del volumen y la producción masiva de información a la que se enfrentaban, el surgimiento de estas máquinas hace que las bibliotecas imaginaran novedosas soluciones, el autor trae en consideración los primeros intentos de recuperación bibliográfica llevados a cabo mediante experimentos en Estados Unidos de la mano de Harley Tillet. Asimismo, describe los primeros sistemas de almacenamiento masivo en disco duro que resultaba de interés para las bibliotecas.

Jesse Shera ${ }^{2}$ no puede dejar de mencionarse cuando de automatización y teorización de la ciencia de la información se refiere, y esta no es la excepción, pues durante varias décadas este bibliotecario estadounidense, como lo indica el Dr. Voutssás influenció en la investigación e innovación en la automatización de bibliotecas desde su trabajo como director de la Escuela de Bibliotecología de la

2 "Ph.D. in Library Science from the Graduate Library School of the University of Chicago in 1944. After working several years at the University of Chicago Library, Shera became dean of the Western Reserve University School of Library (1952-1982), expanding its faculty and adding a doctoral program within few years. In 1952, Shera reorganized the American Documentation Institute (ADI), which eventually became the American Society for Information Scientists. In 1955, in collaboration with James Perry and Alan Kent, Shera established the Center for Documentation and Communications Research (CDRC), which developed a program of teaching and research in the emerging field of information retrieval" http:/ucla245.pbworks.com/w/page/8751414/ Jesse\%20Shera. Fecha de consulta: 10 de Junio de 2020
Western Reserve University en Cleveland, Ohio en 1952 y desde el Centro de Investigaciones en Documentación y Comunicación, fundado en 1955 en compañía de otros profesionales de la bibliotecología como James Perry, Alan Kent; Shera y su equipo orientaron sus investigaciones en los servicios de referencia, clasificación para la organización bibliográfica, procesos de recuperación de información con ayuda de computadoras y otros procesos innovadores para la época. Eventos académicos como simposios, artículos y memorias publicadas dejan evidencia de los importantes aportes realizados por Shera al desarrollo de las Ciencias de la Información.

La tercera parte del texto, hace énfasis en la producción de tarjetas catalográficas. El autor parte del proceso de elaboración de catálogos en tarjetas de cartón y las implicaciones de tiempo para mecanografiarlas así como la imposibilidad de fotocopiarlas para reproducir las necesarias por obra en los años cincuenta, a finales de este periodo se empieza hablar de una máquina xerográfica y con ella la nueva tecnología de fotocopias, aunque, explica que no servía exactamente para las tarjetas catalográficas por el grosor del cartón, sin embargo, se hicieron algunos avances al respecto. Publicaciones en revistas como la "GACETA UNAM" dejan evidencia de que muchas de estas máquinas a disposición de las bibliotecas eran operadas con monedas; así mismo, el autor, describe las técnicas para reproducir documentos a falta de una fotocopiadora, manifiesta que existían desde fines 
del siglo XIX los multigrafos, mimeógrafos, ciclostiles, duplicadores de alcohol y adresógrafos, entre otros, que facilitaban de cierta forma el proceso y lo describe de forma breve.

No obstante, señala que estos procesos resultaban poco eficientes cuando la producción de tarjetas se incrementaba según la capacidad de adquisición, e incluso cuando se trataba de producir tarjetas catalográficas propias y las de otras bibliotecas que lo solicitaran como es el caso de la Biblioteca del Congreso de Estados Unidos, la Biblioteca Británica o de empresas particulares que se dedicaron a la fabricación y venta de juegos de tarjetas para bibliotecas.

Explica el autor, que se exploró la viabilidad de la aplicación de la automatización computarizada a diversos procesos de la biblioteca del Congreso, entre ellas la conversión de las tarjetas catalográficas hacia un formato electrónico, proceso de donde surge MARC (Machine Readable Cataloging) o catalogación legible por máquina; y que de forma similar emprendieron el proyecto en la Biblioteca Británica y colaboraron conjuntamente para desarrollar una nueva versión MARC, producto de ello se reconoció la necesidad y conveniencia de un formato común para intercambiar registros legibles entre los dos países, de ahí surge el estándar MARC II.

Con la necesidad de fabricación y distribución de tarjetas catalográficas se fundaron centros computarizados como el Ohio College Library Center (OCLC) alrededor del año 1967, que se convirtió en un caso exitoso de pro- ducción, distribución de tarjetas catalográficas haciendo uso de computadoras, proyecto que resultó altamente rentable, con el tiempo, esta asociación se transformó en Online Computer Library Center, INC. (OCLC) empresa de tipo privado con participación universitaria.

A Online Computer Library Center, Inc. (OCLC) se le reconoce además por su sistema de recuperación de información bibliográfica en "línea" bajo el concepto del tiempo compartido, la descripción realizada por el Dr. Voutssás, indica que utilizaba una rudimentaria comunicación de equipos a través de una línea telefónica entre una terminal y las bibliotecas asociadas. La Universidad de Stanford desarrolló un proyecto similar que dio origen al sistema "Bibliographic Automation of Large Library Operations using a Time-sharing System" (BALLOTS), posteriormente, cuatro bibliotecas Norteamericanas fundan el "Research Library Group” (RLG) sistema que evolucionó hacia la base de datos bibliográficos en línea "Research $\mathrm{Li}$ braries Information Network” (RLIN). La biblioteca británica también puso a disposición su servicio "British $\mathrm{Li}$ brary Automated Information Service" (BLAISE), y desde Canadá se evidencia la adopción del formato MARC para desarrollar su propia versión generando así su propio sistema de clasificación canadiana, y tras múltiples rediseños finalmente generaron una base de datos denominada DOBIS.

La cuarta parte del texto, muestra un aspecto de gran relevancia que marca 
hasta cierto punto el inicio de lo que hoy conocemos como Ciencias de la Información, el autor se refiere al momento en que surge una necesidad imperante de información especializada en el ámbito científico, en este punto menciona de forma acertada el artículo titulado " $A s$ we may think" de Vannevar Bush publicado en 1945 que resulta para la época un texto de reflexión futurista, Bush fue considerado como un visionario al atreverse a imaginar tecnología que aún no se desarrollaba como las computadoras, la internet, el hipertexto, etc. Sin embargo sus pretensiones de momento responden a la urgente búsqueda de soluciones a problemas que venían siendo protagonistas y que crecían vertiginosamente: la información; su importancia para asuntos de corte científico, político, económico, su acumulación y organización, y su recuperación.

Nuevamente el texto indica que a muchos teóricos y profesionales del campo preocupaba el proceso de recuperación de la información en un vasto universo de producción científica, académica, investigativa, etc; es así como también menciona a otro icono del área, Calvin Mooers ${ }^{3}$ y su término "information retrieval" uno de los es-

3 "Calvin Mooers - Biography, History and Inventions. "Calvin Northrup Mooers (19191994) was an American computer scientist, an influential figure in the early information science community, who had coined the term information retrieval in 1950, and so called Mooers's law in 1959: An information retrieval system will tend not to be used whenever it is more painful and troublesome for a customer to have information than for him not to have it"

https://history-computer.com/Dreamers/Mooers. html. Fecha de consulta: 10 de Junio de 2020 labones primordiales del proceso que condujo a la posterior aparición de las ciencias de la información.

Seguidamente, el texto resulta gratamente enriquecedor y da paso al diálogo y a la discusión, puesto que el autor realiza un análisis sobre las diferentes concepciones de los términos: Documentación, informática, ciencias de la Información, entre otras, considerando la época, el lugar y el contexto; de la interpretación que se hace de los textos disponibles al respecto, comenta el Dr. Voutssás, depende de la definición de estos términos y permite establecer el punto de encuentro entre las bibliotecas y computadoras, esta sinergia, surge entonces con la búsqueda de solventar retos relacionados con procesos de colecta, organización, almacenamiento, recuperación y distribución de grandes cantidades de información científica de forma masiva, precisa, y oportuna.

El autor finaliza este apartado de su publicación con la gestión realizada por México ante esta inminente necesidad de recopilación y distribución de información científica en la década de los cincuenta que los llevó a crear centros de documentación para apoyo a la ciencia y la tecnología en los países en desarrollo; no obstante, decisiones de tipo administrativo provocaron ciertos tropiezos en los procesos adelantados por lo que solo hasta los setenta el gobierno Mexicano prestó atención a la importancia de impulsar el desarrollo y aplicación de la ciencia en México.

En la quinta parte, el autor inicia su disertación refiriéndose al eje central 
de las dos partes abordadas anteriormente, la que corresponde a las tarjetas catalográficas y la importancia de la documentación científica; estos temas de relevante interés en los sistemas bibliotecarios en la segunda mitad del siglo XX hicieron que la Biblioteca del Congreso de Estados Unidos, la National Science Foundation y el Council on Library Resources (CLR), organizaran conferencias para abordar estas temáticas específicas. Las argumentaciones del autor en este capítulo, surgen del análisis de memorias de conferencias que contienen valiosos apuntes sobre los procesos de automatización, almacenamiento magnético y métodos de impresión.

Algunas ponencias presentadas en el evento son reseñadas por el autor y demuestran la existencia de inconvenientes técnicos para los bibliotecarios de los años sesenta, por ejemplo, la impresión solo en mayúsculas, la existencia de un solo tipo de letra, la no existencia de las cursivas, ni negritas, entre otras características.

El autor describe y explica básicamente el porqué de estas deficiencias y para ello hace un recorrido a la evolución del código binario (bit) base de las computadoras electrónicas, la necesidad de los códigos alfanuméricos, la introducción del Binary Coded Decimal Interchange Code (BCDIC) o Código de Intercambio Decimal Codificado en Binario (byte); comenta además cómo y quién propuso el código American Standard Code for Information Interchange (ASCII) o Código Estándar Americano para Intercambio de Información y más adelante el código Extended Binary Coded Decimal Interchange Code (EBCDIC ) o Código Extendido de Intercambio Decimal Codificado en Binario y finaliza su disertación con la versión ASCII que permitió 256 combinaciones.

Otro aspecto que destaca en las fuentes documentales según el autor, corresponde al "tamaño de registro", donde determinar la longitud de cada campo implicaba utilizar una determinada capacidad de almacenamiento. Se ilustra de forma clara en qué consiste el problema partiendo de la construcción básica de un archivo o fichero formado por columnas y renglones (filas) y la definición de una longitud fija para cada campo. Es precisamente esta definición "fija" de longitud la que causaba inconvenientes, puesto que se corría el riesgo de que en algún momento se requiera más espacio del establecido o se desaproveche espacio dejándolo en blanco; estas discusiones dan pie además para referirse al almacenamiento y al costo. Así las cosas, se deduce que en el caso de requerir mayor capacidad el costo también será mayor; con el fin de solucionar problemas como este resolvieron buscar la forma de establecer campos con longitudes variables y utilizar un marcador que permitiera identificar los límites del campo llegando al concepto de "etiquetas numéricas".

El tema de automatización de bibliotecas había causado revuelo, tanto así, que se vieron en la necesidad de convocar nuevas conferencias para discutir estas y otras problemáticas 
específicas y de igual forma se publicaron revistas con un incremento notable de producciones relacionadas con estos temas.

\section{La Automatización de bibliotecas en México}

La sexta parte del texto se centra particularmente en la automatización de bibliotecas en los años sesenta y setenta en México, este apartado permite evidenciar la existencia de sinergia entre bibliotecas y computadoras en referencias documentales provenientes de la "Gaceta UNAM de la Universidad Nacional Autónoma de México", los "Anuarios de Bibliotecología y Archivología" y otros que permiten notar la importancia de los procesos de clasificación automática de documentos, revistas y libros, así como con la búsqueda, recuperación e indizado automáticos de información. Se evidencia también la realización de foros, cursos o conferencias impartidas en México así como la participación de expertos nacionales e internacionales en el tema de recuperación de información y automatización de bibliotecas.

De igual forma, el autor, hace una reseña de la primera tesis en el marco de esta temática en México, así como el surgimiento de la Dirección General de Bibliotecas (DGB) de la UNAM y los proyectos y eventos subsecuentes que marcaron el desarrollo de los procesos de automatización, enfrentándose a continuos retos de costos, peso, velocidad y volumen.

Se permite también prestar aten- ción a la participación del gobierno Mexicano en la búsqueda de fomentar la ciencia y la tecnología en su país y la creciente necesidad de crear un Sistema Nacional de Información Científica y Tecnológica, es así como, considerando que la UNAM tenía un buen porcentaje de infraestructura de investigación en el país, estimuló su desarrollo y se crea en 1971 el Centro de Información Científica y Humanística (CICH) de la UNAM cuyo objetivo era prestar servicios de información especializada, el texto permite hacerse una idea de los equipos y la tecnología utilizada en la época, hace una descripción del tipo de computadoras, impresoras e incluso se detiene a explicar el funcionamiento interno del componente fundamental de las computadoras como lo es la memorias RAM y su importancia histórica.

Los análisis realizados en torno al anuario estadístico de la UNAM demuestran la importancia de la información en la institución y evidencia el rápido crecimiento de volúmenes en las bibliotecas, el autor hace un recorrido sobre el contenido disponible en los diferentes informes, los cuales tienen detalles de la cantidad de libros adquiridos, catalogados y entregados a disposición de los usuarios, de igual forma, destaca en su reseña el momento en que la UNAM otorgó el estatus académico al bibliotecario y de la conformación de un equipo interdisciplinario que permite el surgimiento del proyecto LIBRUNAM 'Sistema Integral de Información y Automatización del Sistema de Bibliotecas de la UNAM', como sistema 
propio, que pronto se convertiría en los cimientos de la creación de bases de datos bibliográficas en México y en América Latina. No obstante como lo describe el autor quien participó personalmente en el proceso, los retos a los que se enfrentaba eran constantes, sus líneas, permiten hacerse una idea de ellos y cómo lograron enfrentarlos hasta que LIBRUNAM cumpliera sus objetivos para fines de 1979 cuando el sistema además de facilitar los procesos técnicos de catalogación permite además entre otras funciones, realizar procesos de búsqueda y recuperación de información muy avanzados para su época, de forma consecuente asumió nuevos retos: "compartir su banco de datos con otras bibliotecas tanto mexicanas como en Latinoamérica".

Las fichas ya catalogadas que disponía LIBRUNAM resultaban ser recursos valiosos para otras instituciones que no contaban con el personal profesional suficiente para catalogación permitiendo así la disminución de tiempo y costo. El reto esta vez era cómo distribuirlo, para la época no existían dispositivos como el CD-ROM ni mucho menos internet, razón por la cual, el autor describe cómo lo hicieron y porque las microfichas fueron una de las opciones más eficientes.

Con el paso del tiempo, surgió un reto adicional que consistía en la estandarización y depuración del catálogo, como es natural el banco de datos que habían exitosamente terminado después de varios años, tenía errores ortográficos, de digitación, de utilización de diferentes estándares en los registros, entre otros; por tanto, se requería la depuración y normalización de la información, razón por la cual se inició con las debidas precauciones la corrección de la base de datos y la normalización de la misma.

Más adelante, el autor, cita a Martínez y Guerrero quienes publicaron el artículo titulado "El Sistema bibliográfico colombiano - Catalogación automatizada - LIBRUNAM" en la Revista Interamericana de Bibliotecología en el año 1980, donde se menciona que el Instituto Colombiano de fomento de la enseñanza superior (ICFES) decidió que LIBRUNAM era la mejor opción para ser utilizada en los sistemas bibliotecarios universitarios colombianos. Queda así demostrada la utilidad de LIBRUNAM y se justifican las razones por las cuales este sistema recibió una importante distinción en México dando origen a nuevos proyectos, entre ellos, la extracción de información estadística de la base de datos como una herramienta de toma de decisiones y conocimiento de tendencias para adquisición de material.

La séptima parte corresponde a la evolución de las telecomunicaciones en México, el autor considera pertinente abordar esta temática para comprender las limitaciones que enfrentaban muchos proyectos de la época. Relata entre varios aspectos, el funcionamiento y las características de los primeros servicios de Telex o teletipo en el año de 1957 que decayeron posteriormente con el auge del fax, posteriormente, explica que con el ánimo de modernizar las telecomunicacio- 
nes y en el marco de los Juegos Olímpicos de 1968, se delegó a Telmex llevar a cabo el proyecto nacional de telefonía y a principios de los setenta se incursionó en el uso de teleproceso, a través de sus líneas, el Dr. Voutssás explica su funcionamiento y además permite observar que la regulación estatal influyó en el uso y el desarrollo de estas tecnologías. Como abrebocas a la llegada del internet a México, el autor, describe brevemente su origen y desarrollo, sus características y novedades. Para 1988 la UNAM y el ITESM se conecta a NSFNET y con ello se da el arribo de internet a México para fines de investigaciones y docencia.

Hasta el momento es posible asumir que el autor centra su disertación en virtud de la automatización de bibliotecas ubicando su eje de atención en la UNAM sin embargo otras instituciones hicieron lo suyo en los años setenta gracias al interés y curiosidad del tema, es así como en la octava parte del texto, menciona evidencias de dicho interés y proyectos de automatización provenientes de la Universidad de Yucatán, la Universidad Autónoma Agraria Antonio Narro, el Instituto Nacional de Investigaciones Agrícolas, el Banco de México, entre otras.

Seguidamente, en la parte novena del texto, el autor a raíz del estudio de las memorias de eventos dedicados expresamente a la automatización de bibliotecas en México en los años ochenta, entre ellos el denominado "Seminario Automatización 81: Las bibliotecas" a cargo de la Asociación de Bibliotecarios de Instituciones de
Enseñanza Superior e Investigación (ABIESI ), presenta de forma breve las iniciativas expuestas a través de ponencias de cohorte teórico y otras de cohorte práctico que permiten observar los avances en cuanto a equipos, programas y telecomunicaciones. Destaca y describe la presentación de David Ramos quien realizó un estudio sobre automatización de bibliotecas en México. Describe también que en este evento se presentó el "correo electrónico" y permite hacerse una idea de su uso y funcionamiento en la época, y reseña cómo fue su desarrollo y evolución. Resalta de igual forma la experiencia presentada en el evento sobre el uso del servicio de OCLC en la biblioteca de la Universidad Iberoamericana.

De igual forma, hace referencia a los proyectos e iniciativas presentados en otros eventos durante los años ochenta, como por ejemplo, la construcción de la base de datos de tesis de la Universidad Autónoma de México-TESIUNAM, la reflexión sobre la construcción de una red automatizada de bibliotecas en esta casa de estudio en forma de estrella que contemplaba microcomputadoras y más adelante ya se reflexionaba sobre las microcomputadoras para uso en las bibliotecas, describe de forma detallada las características en sus diferentes versiones y su uso en las bibliotecas.

Más adelante se refiere al Catálogo Colectivo de Publicaciones Seriadas, que resulta ser, según sus fuentes un tema de discusión relevante. Se refiere además al diseño de un sistema au- 
tomatizado descentralizado de información bibliográfica para las bibliotecas de la UNAM a partir del sistema LIBRUNAM. Éste resulta ser el primer diseño de una red de bibliotecas que ya contempla microcomputadoras en lugar de terminales, de esta manera, continúa la descripción de los proyectos de automatización con sistemas que tuvieron lugar en distintos lugares de México presentando tecnologías y avances novedosos para la época.

La revisión documental que hace el autor de los eventos organizados por instituciones académicas y gubernamentales permite seguir el avance de la automatización de bibliotecas en México en la década de los ochenta, el autor comenta además sobre la evidencia de la inclusión del código de barras y lectores laser para procesos de préstamo en la biblioteca central de la UNAM para el año de 1986, que causó fascinación entre los usuarios y claramente una necesidad incipiente de capacitación para el personal de las bibliotecas. Además el CD-ROM resulta ser la solución para los problemas de distribución de bancos bibliográficos y el autor del texto destaca su importancia en este objetivo.

La última parte teórica del texto, referida a la automatización de bibliotecas en México en los años noventa, reivindica la importancia que tuvo la información distribuida por CD ROM, los avances en el desarrollo de las telecomunicaciones, internet y la llegada de las bibliotecas digitales.

El autor, a través del texto sugiere una división de vertientes a partir de la llegada de la red, una que llevó por el camino a los continuos avances en el campo de desarrollo de procesos de automatización en cuanto a procesos administrativos a bien saber: adquisición, procesos técnicos, inventarios, préstamo, circulación, suscripciones etc. y otra, a la necesidad de crear colecciones y servicios en red, cuestión que más adelante derivaría en el concepto de "bibliotecas digitales".

Claramente los costos de los recursos tecnológicos utilizados fueron disminuyendo y los modelos de lectores de CD-ROM incrementaron tanto capacidad como velocidad, sin embargo en la descripción que hace el autor, la expansión de la web causó el declive de esta tecnología. En este punto se fortalece el uso de catálogos computarizados disponibles al público en la UNAM y la culminación de los grandes catálogos en tarjetas de cartón pese a que había quienes defendían la tradición pero no hubo marcha atrás. Bibliotecas como la del Congreso de Estados Unidos había cerrado la actualización de su catálogo en tarjetas desde 1980 y el mayor productor de tarjetas del mundo OCLC, llevaba años consecutivos a la baja en su producción anual de tarjetas.

Este apartado del texto contiene detalladamente avances, proyectos e iniciativas de los años noventa que evidencian el vertiginoso desarrollo de los procesos de automatización en las bibliotecas de México, es así que tanto bibliotecarios como usuarios debieron acostumbrarse a los cambios pues poco 
a poco la edición en papel disminuye y los recursos en línea toman fuerza. En esta época surgen importantes y reconocidas bases de datos que aún están vigentes: Biblat, Clase, Latindex, entre otras. Por su parte, las revistas académicas de la época de manera gradual disminuyen su edición en papel, muchas de ellas se encontraban disponibles en línea o electrónicamente.

Nuevamente el Dr. Voutssás se detiene a conceptualizar términos puesto que las condiciones han cambiado, hasta este momento de la historia se entendía que todos los proyectos que involucraron a bibliotecas con computadoras o redes se denominaban de "automatización”, sin embargo, el término de "biblioteca digital" empieza a gestar concepciones diferentes.

El concepto de "biblioteca digital" entonces considera a todos aquellos "proyectos y sistemas relacionados con el desarrollo de colecciones, servicios, repositorios, bancos de datos, etcétera, en la web" (Voutssás Márquez, 2019, pág. 407) , por su parte, el término "automatización de bibliotecas" describe aquellos "proyectos, paquetes y sistemas computacionales que ayudan a las bibliotecas en sus procesos y actividades administrativas: adquisiciones, proceso técnico, inventarios, préstamo, etcétera” (Voutssás Márquez, 2019, pág. 407). Según las fuentes del autor, para la segunda mitad de la década de los noventa, LIBRUNAM, TESIUNAM y SERIUNAM ya están disponibles al público vía web.

Para finalizar, el Dr. Voutssás, con- cluye de manera acertada y retomando los aspectos más relevantes de su disertación, en el ambiente deja una sensación de lo mal que se han interpretado los conceptos, "para bien o para mal" como él lo dice, la tecnología ha estado íntimamente ligada a la biblioteca, pero el resultado de esta unión depende del punto de vista en que se observe y actúe ante estas dinámicas; con el tiempo se ha olvidado lo que realmente importa en esta sinergia, las colecciones.

\section{Conclusiones}

Como se ha visto, en la actualidad, la inmensa mayoría de revistas académicas están disponibles en línea y gradualmente dejaron de editarse en papel, este y otros cambios semejantes sólo han sido posibles gracias al esfuerzo interdisciplinario del talento humano que ha visto una oportunidad de mejora y eficiencia con la ayuda de herramientas tecnológicas. Es sabido que a medida que la información viaja entre tantas formas de interacción se requiere contar con medios mucho más eficientes y efectivos de recolección, tratamiento y almacenamiento de la información; los términos Big Data, inteligencia artificial ya no deben ser conceptos ajenos a las actuales realidades sino más bien que se deben ir incorporando a la realidad social de la humanidad.

Las fuentes bibliográficas utilizadas, la forma de abordar la temática, el nivel de detalle de ejemplos y apreciaciones, la utilización de imágenes representativas, hacen que el texto del 
Dr. Voutssás resulte ser agradable, indispensable y de gran interés para el campo de la bibliotecología y de las Ciencias de información que aún están en proceso de construcción.

Esta obra es una invitación a la reflexión, no sólo de conceptos para entender el panorama al que se han enfrentado las bibliotecas, si no para entender el futuro al que hay que enfrentarse; la tecnología para muchos está marcando el fin de las bibliotecas, sin embargo, esta es solo una in- genua apreciación; las bibliotecas le han dado vida a la tecnología y los nuevos retos corresponden al aprovechamiento de los avances tecnológicos para administrar la información.

Después de todo, la información es el activo vital para la construcción de conocimiento, su administración y su soporte sea físico o digital nunca dejará de existir, persistirá como base de nuevos conocimientos que la sociedad requerirá para sobrevivir y progresar.

\section{Referencias}

Voutssás Márquez, J. (2019). Los inicios de la automatización de bibliotecas en México. Colección Tecnologías de la Información Instituto de Investigaciones Bibliotecológicas y de la Información Universidad Nacional Autónoma de México 2019. 522 p. Disponible en línea: http://iibi.unam.mx/voutssasmt/documentos/inicios_automatizacion.pdf

\section{Bibliografía}

Bush, V. (1945). As we may think. The atlantic monthly, 176(1), 101-108. Disponible en línea: https:/www.ias.ac.in/article/fulltext/reso/005/11/0094-0103. Fecha de consulta: 10 junio 2020.

Instituto de Investigaciones Bibliotecológicas y de la información (IIBI). Glosa curricular. Juan Voutssás Márquez. Universidad Nacional Autónoma de México. Disponible en: http:// iibi.unam.mx/voutssasmt/voutssas_principal.html. Fecha de consulta: 10 junio 2020

Voutssás Márquez.Universidad Nacional Autónoma de México. Disponible en: http://iibi. unam.mx/voutssasmt/voutssas_principal.html. Fecha de consulta: 10 junio 2020

University of California (UCLA). Information Access reference services. Jesse Shera. Disponible en línea: http://ucla245.pbworks.com/w/page/8751414/Jesse\%20Shera. Fecha de consulta: 10 junio 2020

History-Computer.com. Calvin Mooers. Disponible en: https:/history-computer.com/Dreamers/Mooers.html. Fecha de consulta: 10 junio 2020 